\title{
Skinning and blending with rational envelope surfaces
}

\author{
Michal Bizzarrii ${ }^{* a}$, Miroslav Lávička ${ }^{\mathrm{b}, \mathrm{a}}$, Jiří Kosinka ${ }^{\mathrm{c}}$ \\ ${ }^{a}$ NTIS-New Technologies for the Information Society, University of West Bohemia, Univerzitni 8 , 306 14 Plzeñ, Czech Republic \\ ${ }^{b}$ Department of Mathematics, University of West Bohemia, Univerzitni 8, 30614 Plzeñ, Czech Republic \\ ${ }^{c}$ Johann Bernoulli Institute, University of Groningen, Nijenborgh 9, 9747 AG Groningen, The Netherlands
}

\begin{abstract}
We continue the study of rational envelope (RE) surfaces. Although these surfaces are parametrized with the help of square roots, when considering an RE patch as the medial surface transform in $4 \mathrm{D}$ of a spatial domain it yields a rational parametrization of the domain's boundary, i.e., the envelope of the corresponding 2-parameter family of spheres. We formulate efficient algorithms for $G^{1}$ data interpolation using RE surfaces and apply the developed methods to rational skinning and blending of sets of spheres and cones/cylinders, respectively. Our results are demonstrated on several computed examples of skins and blends with rational parametrizations.
\end{abstract}

Key words: Rational envelope surface, medial axis transform, blending, skinning

\section{Introduction}

One of the main issues when dealing and computing with geometric objects is the choice of a suitable type of representation [1]. This is important not only from the point of view of geometry representation itself, but also for formulating subsequent and downstream algorithms. A special role is played by parametric representations as these provide easy generation of points on curves and surfaces, and are well suited for surface rendering, computing transformations, determining offsets, admit simple curvature computation, and play a key role in various intersection problems. For these reasons, parametric descriptions are employed in computer graphics and also in computeraided (geometric) design. Among all parametrizations, the most important ones are those that can be described with the help of (piece-wise) polynomial or rational functions, forming the basis of standard CAD systems as the so-called NURBS objects. The NURBS representation (where NURBS stands for №n- $\underline{\text { Uniform }}$ Rational $\underline{B}-$ Spline) is considered the universal standard in technical practice, offering a unifying data exchange format and being able to exactly represent, for example, conics, quadrics, and many other elementary geometric objects from technical applications [2], including free-form spline curves and surfaces.

On the other hand, many natural geometric operations applied to NURBS curves or surfaces do not preserve the rationality of the derived objects. Among the most frequent of such operations are offsetting, the operation of convolution, and the construction of envelopes and

\footnotetext{
* Corresponding author

Email addresses: bizzarri@ntis.zcu.cz (Michal Bizzarri), lavicka@kma.zcu.cz (Miroslav Lávička), J.Kosinka@rug.nl (Jiří Kosinka)
}

Minkowski sums. Hence, studying derived or transformed object rationality belongs to challenging problems in geometric modelling [3, 4]. Nonetheless, we consider a more general viewpoint: When the sought-after object should be rational (i.e., representable by a rational parametrization), it is often convenient to use intermediate non-rational representations. The simplest non-rational parametrizations are square-root parametrizations of curves and surfaces. A curve or surface is called square-root parametrizable if it can be rationally parametrized in terms of $t$ or $(u, v)$ and $\sqrt{P(t)}$ or $\sqrt{P(u, v)}$ with polynomial $P(t)$ or $P(u, v)$, respectively.

It is known that any curve given by a square-root parametrization is rational, elliptic, or hyper-elliptic [5]. We exemplify here several constructions based on elliptic or hyper-elliptic curves that lead to rational derived objects. For instance, it has been proved that canal surfaces determined by a rational trajectory of moving spheres and a square-root radius function are rational [6]. Hence, by allowing square-roots in the parametrizations of medial axis transforms, a larger class of rational canal surfaces can be constructed. A similar result holds also for rational ringed surfaces given by a square-root radius function [7]. And recently, the so-called RE curves, i.e., curves yielding rational envelopes, have been introduced [8]. RE curves, although containing square roots, yield rational envelopes and can be constructed by simpler methods than those for Minkowski Pythagorean hodograph curves [9, 10]. They can also be used for canal surface adaptive blending using rational blends.

In this paper we continue the study of [8] and investigate in more detail surface analogies to $R E$ curves. These RE surfaces, considered as medial surface transforms in four-dimensional space $[11,12]$, are parametrized 


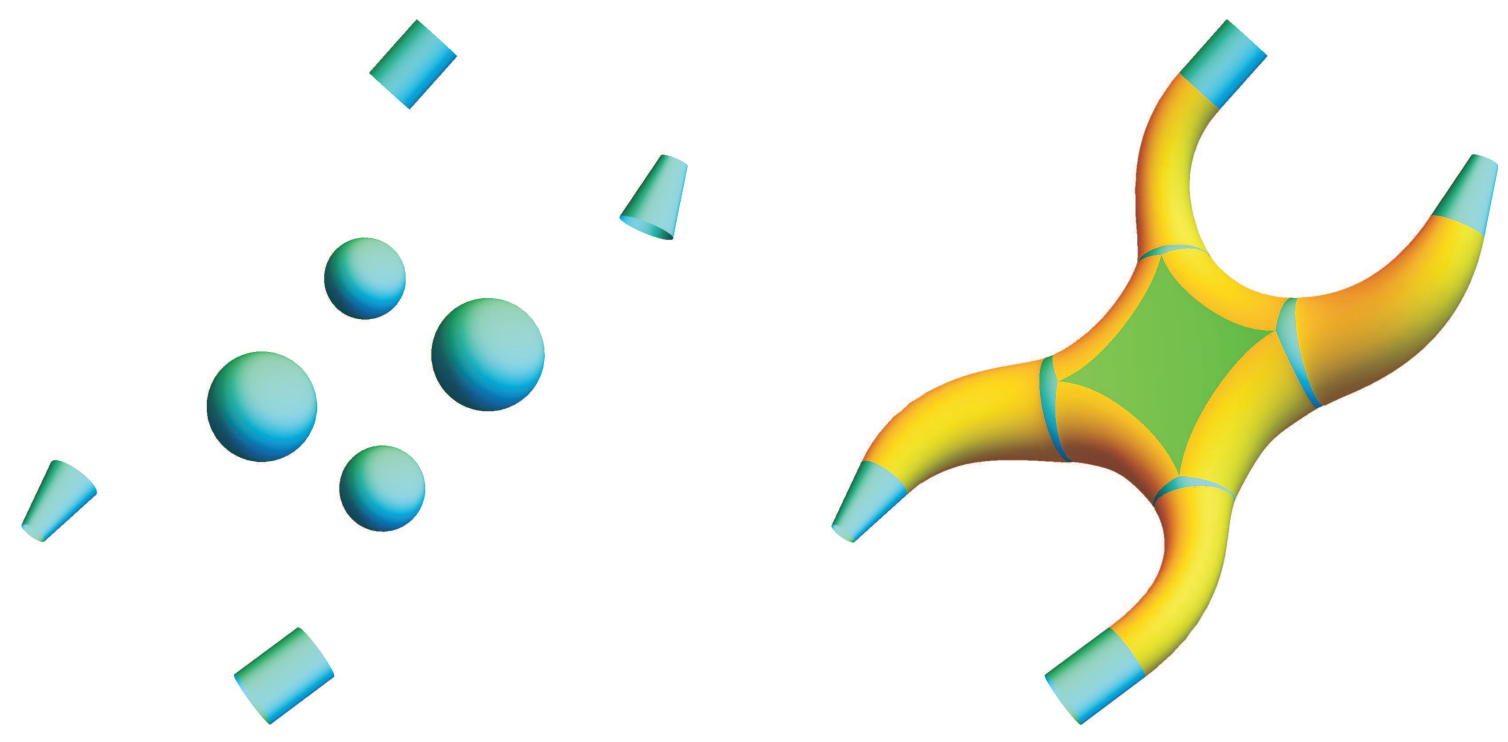

Figure 1: A skin of four spheres combined with blending between spheres and canal surfaces

by square roots. But despite being non-rational, they can be used for constructing rational objects, namely rational envelopes in 3D. We believe that this field still poses many challenges which, when solved, will lead to promising applications. For instance as we show in this paper, rational envelopes of suitable two-parametric systems of spheres have to be considered when branched skins of systems of spheres are computed. As far as we are aware of the literature, these parts of skins/blends have been modelled using non-rational surfaces [13] until now. By utilizing RE surfaces, we offer a simple and novel method for computing rational branched skins of a system of spheres/balls in 3D and rational blends between canal surfaces, including branched blends. Envelopes of one- or two-parametric families of spheres (or balls) play an important role in various applications including computational chemistry, molecular biology, computer animation, and modelling of tubular surfaces. A simple example combining skinning and branching is shown in Fig. 1.

We start by recalling prior knowledge on canal surfaces, skinning and blending, and by defining and investigating RE surfaces (Section 2). This is followed by an interpolation scheme for RE surfaces (Section 3). Our theoretical results are then applied to ball skinning (Section 4) and to canal surface blending (Section 5). Finally, we discuss limitations of our approach (Section 6), and conclude the paper and point out future research directions (Section 7).

\section{Preliminaries}

We recall some fundamental facts about canal surfaces, skinning of balls/spheres, blending between canal surfaces, and rational envelope (RE) surfaces.

\subsection{Canal surfaces}

Consider a one-parameter set of spheres

$$
F(t):|(\mathbf{m}(t)-\mathbf{x})|^{2}-r^{2}(t)=0, \quad t \in I \subseteq \mathbb{R},
$$

where $\mathbf{x}=(x, y, z)$. The envelope $\mathcal{S}$ of $F(t)$ is called a canal surface, the curve $\mathbf{m}(t)$ tracing the centres of the spheres $F(t)$ its spine curve, and the function $r(t)$ describing the radii of $F(t)$ its radius function. By appending the corresponding sphere radii $r(t)$ to the points of the spine curve $\mathbf{m}(t)$ we obtain the medial axis transform (MAT for short). The canal surface $\mathcal{S}$ is thus given by and identified with its medial axis transform $(\mathbf{m}(t), r(t)) \subset \mathbb{R}^{3,1}$, where $\mathbb{R}^{3,1}$ is the 4-dimensional Minkowski space.

It was proved in [14] that any canal surface with a rational spine curve and a rational radius function has a rational parametrization. A technique for computing rational parametrizations of canal surfaces was presented in [15]. We emphasize that although the canal surfaces with rational spine curves and rational radii always possess exact rational parametrizations, approximate parametrization techniques are also investigated in connection with them [16]. This is caused by the computational difficulty of decomposing a rational function into a sum of two squares (SOS) over the real numbers, which is a key ingredient in the parametrization algorithm from [15]. Moreover, there exist rational canal surfaces with square-root parametrized medial axis transforms.

A rational parametrization of a canal surface $\mathcal{S}$ can be easily computed when a rational curve c on $\mathcal{S}$ is known. It is then enough to rotate $\mathbf{c}$ around the tangents of the spine curve $\mathbf{m}$, which gives

$$
\begin{aligned}
& \mathbf{s}(t, u)=\mathbf{m}(t)+ \\
& +\frac{\left(\varrho(u), \mathbf{m}^{\prime}(t)\right) \star(0, \mathbf{c}(t)-\mathbf{m}(t)) \star\left(\varrho(u),-\mathbf{m}^{\prime}(t)\right)}{\left(\varrho(u), \mathbf{m}^{\prime}(t)\right) \star\left(\varrho(u),-\mathbf{m}^{\prime}(t)\right)}
\end{aligned}
$$




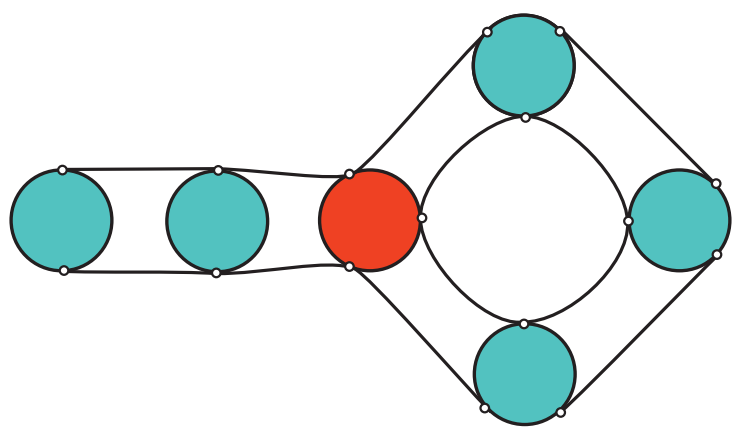

Figure 2: Branching of a skinning surface (2D illustration) on a particular sphere. The red sphere is connected via the skin with 3 neighbouring spheres.

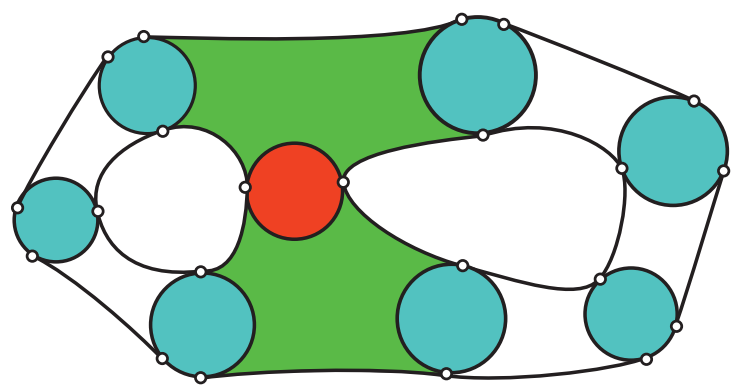

Figure 3: Branching of a skinning surface (2D illustration) when a blend (green) between more than two spheres is needed. The red sphere is connected via the skin with 4 neighbouring spheres.

where $\varrho(u)$ is an arbitrary rational function. The sums $\varrho(u) \pm \mathbf{m}^{\prime}(t)$ of scalars and vectors are considered as quaternions, and $\star$ is the operation of quaternion multiplication; see $[17,18]$ for more details about quaternions.

\subsection{Skinning balls in $3 D$ space}

Skinning is a construction of a $G^{k} / C^{k}$ continuous interpolation curve/surface of an ordered sequence of planar or spatial shapes (most often circles or spheres). It can be viewed as an analogy to the well-known interpolation of point sets, see e.g. [19] and references therein. Due to its technical importance, skinning has attracted the geometric modelling community in recent years and one can find several papers on this topic, see [20, 21, 22, 23]. One of the application areas is computer animation: given a skeletal pose, skinning algorithms are responsible for deforming the geometric skin to respond to the motion of the underlying skeleton.

Following the approach of [21], we consider a given sequence of spheres $\Sigma=\left\{S_{1}, S_{2}, \ldots, S_{n}\right\}$, which is admissible as defined in [24]. Our goal is to describe a $G^{1}$ spline surface $\mathcal{S}(\Sigma)$ skinning this system. As in [24], we allow not only linear sequences of input spheres but also more complicated situations. Especially, we focus on configurations when branched skins should be constructed.

When constructing the skin of spheres in the linear (sub)configuration, $\mathcal{S}(\Sigma)$ consists of the following el- ements: (i) parts of $S_{i}$ obtained as the differences of $S_{i}$ and the spherical caps determined by the contact circles; (ii) surfaces smoothly joining two consecutive spheres $S_{i-1}$ and $S_{i}$ along prescribed contact circles. For constructing the blending shapes (ii) we can use e.g. the algorithm formulated in [25]. It is based on using rational contour curves of canal surfaces for computing their rational parametrizations. It is beyond the scope of this paper to go into details and we refer e.g. to [24].

Nonetheless, the problem is more complicated when branching of skins is allowed. Branching in this case means that there exists a sphere which is connected via the skin with more than two neighbouring spheres; see the red sphere in Figs. 2 and 3. Then two types of situations can arise. Either, branching occurs on a particular sphere, Fig. 2. In this case, the skin consists again of the elements of type (i) and (ii) only. Or, a blend between more than two spheres is needed, giving rise to a new type, type (iii); see the green shapes in Fig. 3. For this reason, the rational envelopes of suitable two-parameter systems of spheres must be investigated in more detail. Note that this problem was partially mentioned already in [24], but non-rational envelopes were applied there.

\subsection{Blending between canal surfaces}

Blending belongs among important operations in Computer-Aided (Geometric) Design and due to its technical importance it has continuously attracted the geometric design community. The main purpose of this operation is to generate one or more surfaces that create a smooth join between the given shapes. Blending surfaces are necessary for rounding edges and corners of mechanical parts, or for smooth connections of separated objects. For an overview of several blending techniques see [19, 3, 13] and the references therein.

The existing approaches to the operation of blending may be classified with respect to the type of surfaces which are used. Parametric blend surfaces are among the most used in applications as they can be easily added to an existing boundary representation of a solid using trimmed surfaces. These blends are defined by specifying contact curves on the given primary surfaces and then computing a blend surface that smoothly connects (or blends) the given surfaces. This works particularly well for blends between two surfaces; the description of multi-sided blends is not so simple. Compared to the parametric case, the use of implicitly defined blend surfaces offers more flexibility for designing blends, since their shape is not restricted to embeddings of their parameter domain. Due to this flexible topology, it is easier to obtain complex, multi-sided blends in the implicit setting.

In addition, when investigating blending surfaces in more detail it is also appropriate to analyse the class of primary surfaces that can be dealt with. Special attention is often paid to blending surfaces between canal surfaces. This topic has been thoroughly studied in recent 


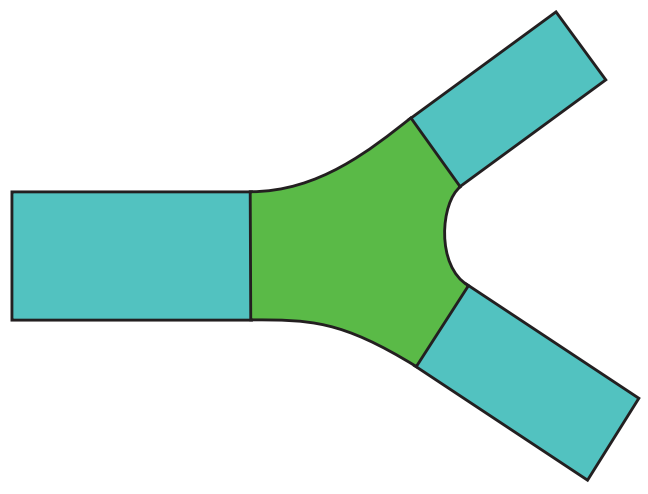

Figure 4: Blending of more than two canal surfaces (2D illustration): 3-way blend.

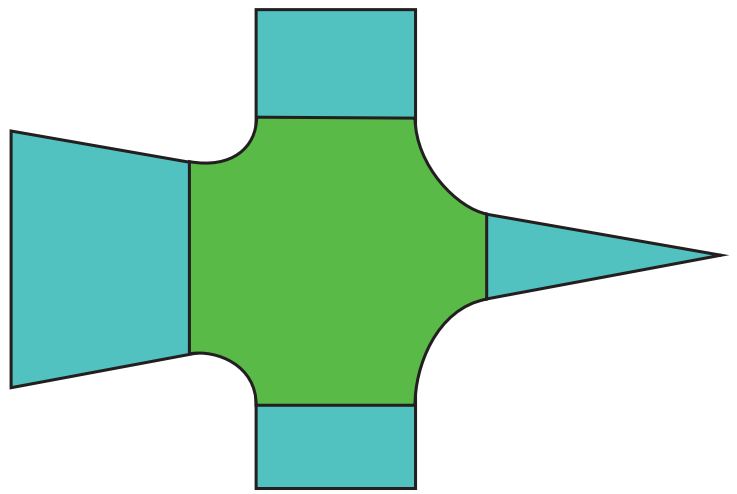

Figure 5: Blending of more than two canal surfaces (2D illustration): 4-way blend (right)

years and one can find many papers devoted to this subject when smooth transitions between two canal surfaces are constructed. In this paper, we focus on the challenging problem of blends between more than two canal surfaces (see Fig. 4 and Fig. 5), and also on the closely related task of constructing branched blends. These types of blend surfaces again consist of the parts which are defined as envelopes of special two-parameter families of spheres (see the green shapes in Fig. 4 and Fig. 5) and a dominant role is played by rational envelopes.

\subsection{Rational envelope surface in $\mathbb{R}^{3,1}$}

Consider a spatial domain $\Omega \subset \mathbb{R}^{3}$ and the family of all inscribed spheres partially ordered with respect to inclusion of the associated balls. An inscribed sphere is called maximal if it is not contained in any other inscribed sphere. Then the medial surface (MS) of $\Omega$ is the locus of all centers $\mathbf{y}$ of maximal inscribed spheres and the medial surface transform (MST) of $\Omega$ is obtained by appending the corresponding sphere radius $r$ to the medial surface, i.e., it consists of the points $(\mathbf{y}, r) \in \mathbb{R}^{3,1}$. The projection

$$
\mathbb{R}^{3,1} \rightarrow \mathbb{R}^{3}: \quad \overline{\mathbf{y}}=(\mathbf{y}, r) \mapsto \mathbf{y}
$$

naturally relates MS to MST.
For a $C^{1}$ segment $\overline{\mathbf{y}}(u, v)=(\mathbf{y}(u, v), r(u, v)) \subset \mathbb{R}^{3,1}$ of an MST, the corresponding boundary of the domain $\Omega$ is given by the envelope formula [11]

$$
\mathbf{x}^{ \pm}=\mathbf{y}-r \mathbf{n}^{ \pm}
$$

where

$$
\begin{array}{r}
\mathbf{n}^{ \pm}=\frac{\left(r_{u} G-r_{v} F\right) \mathbf{y}_{u}+\left(r_{v} E-r_{u} F\right) \mathbf{y}_{v}}{E G-F^{2}} \\
\pm \frac{\left(\mathbf{y}_{u} \times \mathbf{y}_{v}\right) \sqrt{\overline{E G}-\bar{F}^{2}}}{E G-F^{2}}
\end{array}
$$

and $x_{u}$ denotes the partial derivatives of $x$ with respect to $u$, etc. The components $\bar{E}, \bar{F}, \bar{G}$ of the first fundamental form of $\overline{\mathbf{y}}(u, v)$ are computed using the indefinite Minkowski inner product

$$
\langle\mathbf{u}, \mathbf{v}\rangle=u_{1} v_{1}+u_{2} v_{2}+u_{3} v_{3}-u_{4} v_{4},
$$

whereas the components $E, F, G$ of the first fundamental form of $\mathbf{y}(u, v)$ are determined using the standard Euclidean inner product in $\mathbb{R}^{3}$. Singularities and points with reverse boundary orientation (i.e., invalid regions) can be directly detected using the Jacobian of the mapping in (4); see [26] for more details.

The so-called MOS surfaces (i.e., Medial surfaces

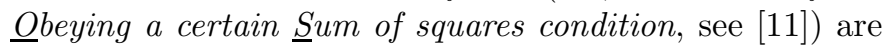
characterized by the condition

$$
\overline{E G}-\bar{F}^{2}=\sigma^{2}(u, v),
$$

where $\sigma(u, v) \in \mathbb{R}(u, v)$. This ensures that the envelope $\mathbf{x}^{ \pm}$is rational. Consequently, $\mathbf{x}^{ \pm}$possesses a normal vector field $\mathbf{n}^{ \pm}=\left(\mathbf{x}^{ \pm}-\mathbf{y}\right) / r$ rationally parametrizing the unit sphere, i.e., $\mathbf{x}^{ \pm}$are rational surfaces with Pythagorean normals (PN surfaces for short); see [27]. Additionally, any rational MOS surface $\overline{\mathbf{y}}$ in $\mathbb{R}^{3,1}$ can be constructed starting from an (associated) rational PN surface $\mathbf{x}$ in $\mathbb{R}^{3}$ and a rational function $r$ in the form

$$
\overline{\mathbf{y}}(u, v)=\left(\mathbf{x}+r \frac{\mathbf{x}_{u} \times \mathbf{x}_{v}}{\left|\mathbf{x}_{u} \times \mathbf{x}_{v}\right|}, r\right) .
$$

We remark that $\mathbf{x}$ will play the role of $\mathbf{x}^{+}$in what follows. However, the main problem that prevents the use of PN and MOS surface in applications is that the algorithms for interpolation with $\mathrm{PN} / \mathrm{MOS}$ surfaces are relatively complicated (as they are based on the dual approach, or on reparametrizations).

Nonetheless, as in the univariate case of RE curves and MATs [8], MOS surfaces are not the only MSTs yielding rational envelopes. Turning back to (4), we only have to guarantee that $r \mathbf{n}^{ \pm}$is rational. This brings us to a broader class of (generally non-rational) RE surfaces, i.e., surfaces

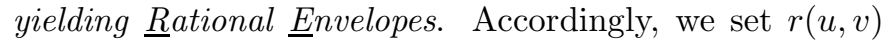
as the square root of some non-negative function $R(u, v)$. This leads to

$$
r r_{u}=\frac{1}{2} R_{u} \in \mathbb{R}(u, v), \quad r r_{v}=\frac{1}{2} R_{v} \in \mathbb{R}(u, v) .
$$



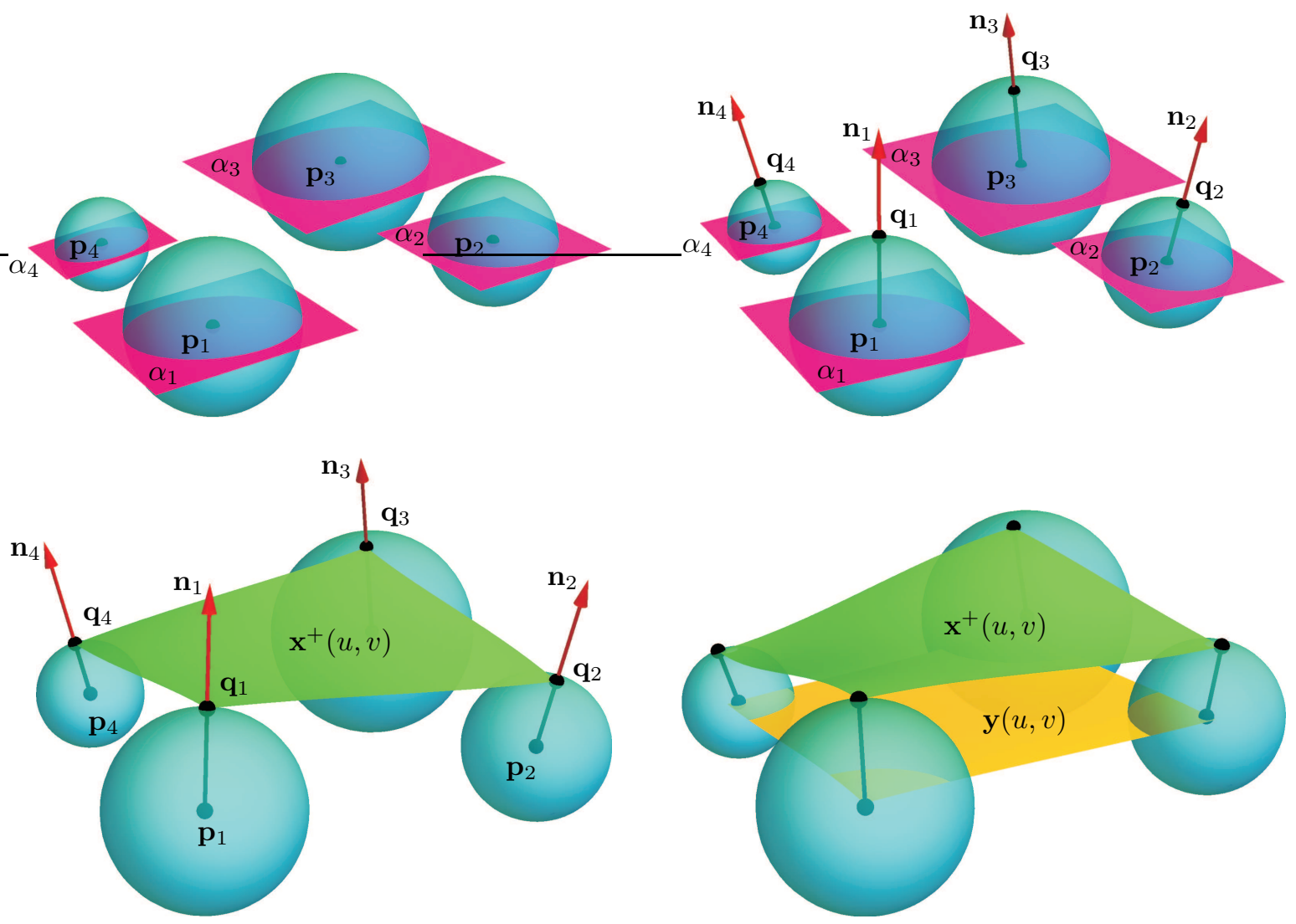

Figure 6: Interpolating input $G^{1}$ data (top left) by an RE surface (yellow, bottom right).

Then the rationality of $r \mathbf{n}^{ \pm}$(and thus also of the envelope $\mathbf{x}^{ \pm}$), cf. (4), is guaranteed by the condition

$$
R\left(\overline{E G}-\bar{F}^{2}\right)=\sigma^{2}(u, v) .
$$

Additionally, any RE surface $\overline{\mathbf{y}}$ in $\mathbb{R}^{3,1}$ can be constructed starting from an (associated) rational surface $\mathbf{x}$ in $\mathbb{R}^{3}$ and a rational function $f$ in the form

$$
\overline{\mathbf{y}}(u, v)=\left(\mathbf{x}+f\left(\mathbf{x}_{u} \times \mathbf{x}_{v}\right), f\left|\mathbf{x}_{u} \times \mathbf{x}_{v}\right|\right) .
$$

In contrast to MOS surfaces, it is easy to generate RE surfaces in the form $\overline{\mathbf{y}}=(\mathbf{y}, r=\sqrt{R})$.

\section{Interpolation by $R E$ surfaces in $\mathbb{R}^{3,1}$}

In this section we introduce a novel method for interpolating points $\overline{\mathbf{p}}_{i}=\left(\mathbf{p}_{i}, r_{i}\right) \in \mathbb{R}^{3,1}, i=1, \ldots, n$ and their associated tangent planes $\bar{\alpha}_{i}$ (or tangent vectors $\overline{\mathbf{t}}_{1 i}$ and $\overline{\mathbf{t}}_{2 i}$ ) by an RE surface. Naturally, this technique creates a close analogy between interpolation of point data sets in $\mathbb{R}^{3,1}$ and the construction of a surface enveloping balls/spheres in $\mathbb{R}^{3}$. The obtained surfaces in $\mathbb{R}^{3,1}$ having rational envelopes in $\mathbb{R}^{3}$ will be consequently used for the construction of parts of branched skins and blends; see Sections 4 and 5, respectively.
The main idea behind the presented method is to start by constructing a polynomial surface $\mathbf{x}(u, v) \subset \mathbb{R}^{3}$ considered as one branch of the envelope (boundary) surface and then to use a suitably chosen function $f(u, v)$ to lift it to $\mathbb{R}^{3,1}$. Thus, we obtain a polynomial MST $\overline{\mathbf{y}}(u, v) \subset \mathbb{R}^{3,1}$, cf. (11), interpolating the given data. Of course the second branch $\mathbf{x}^{-}(u, v)$ of the envelope surface associated to $\overline{\mathbf{y}}(u, v)$ will possess a rational description, too. The main reason for constructing the second branch $\mathbf{x}^{-}(u, v)$ from $\overline{\mathbf{y}}(u, v)$ and not independently is obvious: both branches correspond in parameter, i.e., for a chosen pair $(u, v)$ the corresponding normals intersect at the medial surface. Or put differently, in our approach the MST of the resulting blend is explicitly known and can thus be used for further processing of the blend/skin.

We now show in more detail how the surface $\mathbf{x}(u, v)$ and the function $f(u, v)$ are constructed when some constraints reflecting a particular problem are considered.

\subsection{RE surface patches interpolating given points and as- sociated tangent planes}

Consider $n$ points $\overline{\mathbf{p}}_{i}=\left(\mathbf{p}_{i}, r_{i}\right) \in \mathbb{R}^{3,1}$ and $n$ tangent planes $\bar{\alpha}_{i}$, given by the vectors $\overline{\mathbf{t}}_{i 1}=\left(\mathbf{t}_{i 1}, \tau_{i 1}\right)$ and $\overline{\mathbf{t}}_{i 2}=\left(\mathbf{t}_{i 2}, \tau_{i 2}\right)$; see Fig. 6 (top, left). Using the envelope formula (4), we obtain the associated end points $\mathbf{q}_{i}$ on the 

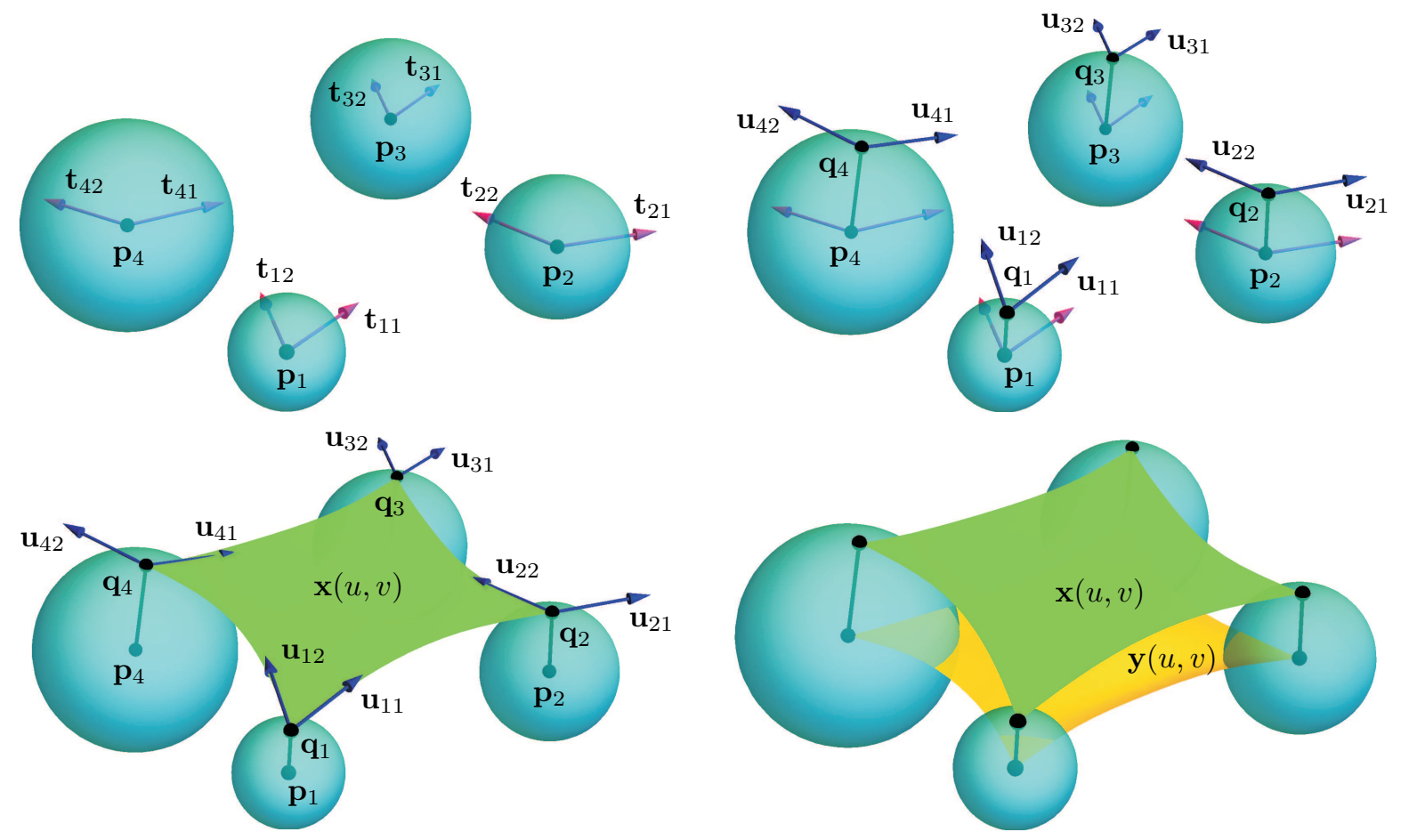

Figure 7: Interpolating $G^{1}$ data (top left) by an RE surface (yellow, bottom right).

corresponding envelope

$$
\begin{array}{r}
\mathbf{q}_{i}=\mathbf{p}_{i}-r_{i} \frac{\left(\tau_{i 1} G_{i}-\tau_{i 2} F_{i}\right) \mathbf{t}_{i 1}+\left(\tau_{i 2} E_{i}-\tau_{i 1} F_{i}\right) \mathbf{t}_{i 2}}{E_{i} G_{i}-F_{i}^{2}} \\
\pm \frac{\left(\mathbf{t}_{i 1} \times \mathbf{t}_{i 2}\right) \sqrt{\bar{E}_{i} \bar{G}_{i}-\bar{F}_{i}^{2}}}{E_{i} G_{i}-F_{i}^{2}}
\end{array}
$$

where $E_{i}=\left|\mathbf{t}_{i 1}\right|^{2}, \quad F_{i}=\left|\mathbf{t}_{i 2}\right|^{2}, \quad G_{i}=\mathbf{t}_{i 1} \cdot \mathbf{t}_{i 2}$ and $\bar{E}_{i}=\left|\mathbf{t}_{i 1}\right|^{2}-\tau_{i 1}^{2}, \bar{F}_{i}=\left|\mathbf{t}_{i 2}\right|^{2}-\tau_{i 2}^{2}, \bar{G}_{i}=\mathbf{t}_{i 1} \cdot \mathbf{t}_{i 2}-\tau_{i 1} \tau_{i 2}$. Naturally, the normal vectors $\mathbf{n}_{i}$ of the envelope surface at $\mathbf{q}_{i}$ are

$$
\mathbf{n}_{i}=\lambda_{i}\left(\mathbf{q}_{i}-\mathbf{p}_{i}\right), \quad \lambda_{i} \in \mathbb{R}
$$

see Fig. 6 (top, right). We can construct a polynomial patch $\mathbf{x}(u, v)$ interpolating the points $\mathbf{q}_{i}$ and the associated normal vectors $\mathbf{n}_{i}$; see Fig. 6 (bottom, left). Next, using formula (11) we lift $\mathbf{x}(u, v)$ to $\mathbb{R}^{3,1}$ and as a result we arrive at the MST $\overline{\mathbf{y}}(u, v)$. Conditions on $\overline{\mathbf{y}}(u, v)$ to interpolate the points $\overline{\mathbf{p}}_{i}$, i.e.,

$$
\overline{\mathbf{y}}\left(u_{i}, v_{i}\right)=\overline{\mathbf{p}}_{i}
$$

yield the following conditions on the function $f(u, v)$ :

$$
f_{i}\left(u_{i}, v_{i}\right)=\frac{r_{i}}{\left|\mathbf{n}_{i}\right|}
$$

Moreover, we require that $\overline{\mathbf{y}}(u, v)$ touches the tangent planes $\bar{\alpha}_{i}$ at the given points $\overline{\mathbf{p}}_{i}$, i.e.,

$$
\begin{aligned}
& \overline{\mathbf{y}}_{u}\left(u_{i}, v_{i}\right)=\beta_{i 1} \overline{\mathbf{t}}_{i 1}+\beta_{i 2} \overline{\mathbf{t}}_{i 2}, \\
& \overline{\mathbf{y}}_{v}\left(u_{i}, v_{i}\right)=\gamma_{i 1} \overline{\mathbf{t}}_{i 1}+\gamma_{i 2} \overline{\mathbf{t}}_{i 2},
\end{aligned}
$$

which (for each of these identities) forms a system of four linear equations for three variables $f_{u}, \beta_{i 1}, \beta_{i 2}$ and $f_{v}, \gamma_{i 1}, \gamma_{i 2}$, respectively. However, the equations in each system are dependent, which follows from the next dependency conditions:

$$
\left\langle\widetilde{\mathbf{n}}, \overline{\mathbf{y}}_{u}\right\rangle=0, \quad\left\langle\widetilde{\mathbf{n}}, \overline{\mathbf{y}}_{v}\right\rangle=0
$$

where $\widetilde{\mathbf{n}}=(\mathbf{n},|\mathbf{n}|)=\left(\mathbf{x}_{u} \times \mathbf{x}_{v},\left|\mathbf{x}_{u} \times \mathbf{x}_{v}\right|\right)$ is an isotropic vector; see [28] for more details. Thus we can choose three arbitrary equations in each system, e.g., the first three of them. Thus we solve

$$
\begin{aligned}
& \mathbf{y}_{u}\left(u_{i}, v_{i}\right) \cdot \mathbf{m}_{i}=0 \\
& \mathbf{y}_{v}\left(u_{i}, v_{i}\right) \cdot \mathbf{m}_{i}=0
\end{aligned}
$$

where $\mathbf{m}_{i}=\mathbf{t}_{i 1} \times \mathbf{t}_{i 2}$. This yields

$$
\begin{aligned}
& f_{u}^{i}\left(u_{i}, v_{i}\right)=-\frac{\left(\mathbf{x}_{u}\left(u_{i}, v_{i}\right)+f_{i}\left(u_{i}, v_{i}\right) \mathbf{n}_{u}\left(u_{i}, v_{i}\right)\right) \cdot \mathbf{m}_{i}}{\mathbf{n}_{i} \cdot \mathbf{m}_{i}} \\
& f_{v}^{i}\left(u_{i}, v_{i}\right)=-\frac{\left(\mathbf{x}_{v}\left(u_{i}, v_{i}\right)+f_{i}\left(u_{i}, v_{i}\right) \mathbf{n}_{v}\left(u_{i}, v_{i}\right)\right) \cdot \mathbf{m}_{i}}{\mathbf{n}_{i} \cdot \mathbf{m}_{i}}
\end{aligned}
$$

For valid input data, $\mathbf{n}_{i} \cdot \mathbf{m}_{i}$ does not vanish and thus one always obtains a solution, cf. [26]. Hence when constructing a rational function $f(u, v)$ satisfying conditions (15) and (19), the envelope surface associated to $\overline{\mathbf{y}}(u, v)$ smoothly joins the given $n$ spheres at the prescribed points and respects the given tangent planes; see Fig. 6 (bottom, right). 


\subsection{RE surface patches interpolating given points with} boundaries interpolating prescribed tangent directions

In this section we show how $n$ points $\overline{\mathbf{p}}_{i}$ and $2 n$ tangent vectors $\overline{\mathbf{t}}_{i 1}$ and $\overline{\mathbf{t}}_{i 2}$ at $\overline{\mathbf{p}}_{i}$, see Fig. 7 (top, left), can be interpolated by a surface yielding rational envelopes. The whole construction is based only on solving a system of linear equations. Again, we start by computing the corresponding points $\mathbf{q}_{i}$ and normal vectors $\mathbf{n}_{i}$, cf. formulae (12) and (13). Then we choose tangent vectors $\mathbf{u}_{i 1}$ and $\mathbf{u}_{i 2}$ at $\mathbf{q}_{i}$, e.g. by projecting $\mathbf{t}_{i j}, j=1,2$ to the plane given by the point $\mathbf{q}_{i}$ and the normal vector $\mathbf{n}_{i}$, i.e.,

$$
\mathbf{u}_{i j}=\mathbf{t}_{i j}-\frac{\mathbf{t}_{i j} \cdot \mathbf{n}_{i}}{\left|\mathbf{n}_{i}\right|^{2}} \mathbf{n}_{i}
$$

see Fig. 7 (top, right). The partial derivatives of the medial surface $\mathbf{y}(u, v)$, cf. (11),

$$
\begin{aligned}
& \mathbf{y}_{u}=\mathbf{x}_{u}+f_{u}\left(\mathbf{x}_{u} \times \mathbf{x}_{v}\right)+f\left(\mathbf{x}_{u u} \times \mathbf{x}_{v}\right)+f\left(\mathbf{x}_{u} \times \mathbf{x}_{v u}\right) \\
& \mathbf{y}_{v}=\mathbf{x}_{v}+f_{v}\left(\mathbf{x}_{u} \times \mathbf{x}_{v}\right)+f\left(\mathbf{x}_{u v} \times \mathbf{x}_{v}\right)+f\left(\mathbf{x}_{u} \times \mathbf{x}_{v v}\right)
\end{aligned}
$$

imply that the second partial derivatives $\mathbf{x}_{u u}$ and $\mathbf{x}_{v v}$ of $\mathbf{x}$ have to be in this case suitably chosen to ensure the continuity conditions

$$
\begin{array}{ll}
\overline{\mathbf{y}}\left(u_{i}, v_{i}\right) & =\overline{\mathbf{p}}_{i}, \\
\overline{\mathbf{y}}_{u}\left(u_{i}, v_{i}\right) & =\beta_{i} \overline{\mathbf{t}}_{i 1}, \\
\overline{\mathbf{y}}_{v}\left(u_{i}, v_{i}\right) & =\gamma_{i} \overline{\mathbf{t}}_{i 2},
\end{array}
$$

where $\beta_{i}, \gamma_{i} \in \mathbb{R}$. Of course, the values $f_{i}=f\left(u_{i}, v_{i}\right)$ can be computed analogously as in the previous section, cf. (15),

$$
f\left(u_{i}, v_{i}\right)=\frac{r_{i}}{\left|\mathbf{u}_{i 1} \times \mathbf{u}_{i 2}\right|} .
$$

To summarize, the values of $\mathbf{q}_{i}, \mathbf{u}_{i 1}, \mathbf{u}_{i 2}$ and $f_{i}=$ $f\left(u_{i}, v_{i}\right)$ are known and the values of $f_{u}^{i}=f_{u}\left(u_{i}, v_{i}\right)$, $f_{v}^{i}=f_{v}\left(u_{i}, v_{i}\right), \mathbf{v}_{i 1}=\mathbf{x}_{u u}\left(u_{i}, v_{i}\right), \mathbf{v}_{i 2}=\mathbf{x}_{v v}\left(u_{i}, v_{i}\right)$ and $\beta_{i}, \gamma_{i}$ shall be computed to satisfy conditions (22). Since it is enough to choose arbitrary three equations for each identity (22), cf. (17), we solve for each $i \in\{1, \ldots, n\}$ the following two systems of three linear equations:

$$
\begin{array}{r}
\mathbf{u}_{i 1}+f_{u}^{i}\left(\mathbf{u}_{i 1} \times \mathbf{u}_{i 2}\right)+f_{i}\left(\mathbf{v}_{i 1} \times \mathbf{u}_{i 2}\right) \\
+f_{i}\left(\mathbf{u}_{i 1} \times \mathbf{x}_{u v}\left(u_{i}, v_{i}\right)\right)=\beta_{i} \mathbf{t}_{i 1} ; \\
\mathbf{u}_{i 2}+f_{v}^{i}\left(\mathbf{u}_{i 1} \times \mathbf{u}_{i 2}\right)+f_{i}\left(\mathbf{x}_{u v}\left(u_{i}, v_{i}\right) \times \mathbf{u}_{i 2}\right) \\
+f_{i}\left(\mathbf{u}_{i 1} \times \mathbf{v}_{i 2}\right)=\gamma_{i} \mathbf{t}_{i 2} .
\end{array}
$$

These systems possess a solution if and only if it is guaranteed that $\mathbf{u}_{i 1} \times \mathbf{u}_{i 2} \neq \mathbf{0}$ and $\mathbf{u}_{i 2} \cdot \mathbf{t}_{i 1} \neq 0, \mathbf{u}_{i 1} \cdot \mathbf{t}_{i 2} \neq 0$. And this is always satisfied for valid input data; see [26]. Consequently, the solution of the linear systems (24) and (25) gives the envelope surface $\mathbf{x}$; see Fig. 7 (bottom, left). Finally, constructing a function $f$ that interpolates (23) and the solution of (24) and (25) and lifting the patch $\mathbf{x}$ to $\mathbb{R}^{3,1}$, cf. (11), we obtain a parametrization of the RE surface patch $\overline{\mathbf{y}}$ interpolating the points $\overline{\mathbf{p}}_{i}$ and the tangent directions $\overline{\mathbf{t}}_{i 1}$ and $\overline{\mathbf{t}}_{i 2}$ at $\overline{\mathbf{p}}_{i}$ as required; see Fig. 7 (bottom, right).
Remark 3.1. Solving the linear systems (24) and (25) yields a $2 \times 2 n$-parametric solution, in general. In addition, we can also leave the lengths of the tangent vectors $\mathbf{u}_{i j}$ and the values of the second mixed derivatives $\mathbf{x}_{u v}\left(u_{i}, v_{i}\right)$ of $\mathbf{x}(u, v)$ as free parameters and solve $n$ systems of six equations yielding a $9 n$-parametric solution. The free parameters can be used for modelling purposes, i.e., the most suitable solution can be chosen such that the resulting surface satisfies further criteria. For instance, we can require that the radii of the inscribed spheres of the envelope change as minimally as possible. Thus, we minimize the objective function

$$
\Phi=\int_{0}^{1} \int_{0}^{1}\left(r_{u}^{2}+r_{v}^{2}\right) \mathrm{d} u \mathrm{~d} v .
$$

For minimizing (26) one can use e.g. the classical Newton's method which is characterized by fast convergence; see e.g. $[29,30,31]$.

\section{Skinning balls using rational envelope surfaces}

Given $n$ spheres in $\mathbb{R}^{3}$, our goal is to construct a rational skinning surface which smoothly envelopes these given spheres; see Fig. 8. The main idea of our approach is to transform the problem to $\mathbb{R}^{3,1}$ and construct an RE surface passing through the prescribed points and touching planes in $\mathbb{R}^{3,1}$. We use the method described in Section 3.1. The corresponding envelope surface in $\mathbb{R}^{3}$ then yields the two main elements of the desired skinning surface (besides the parts of canal surfaces on the edges).

Let the spheres $\mathcal{S}_{i}$ be given by the points $\overline{\mathbf{p}}_{i}=\left(\mathbf{p}_{i}, r_{i}\right) \in$ $\mathbb{R}^{3,1}, i=1, \ldots, n$, such that for each $\overline{\mathbf{p}}_{i}$ the two points $\overline{\mathbf{p}}_{i-1}, \overline{\mathbf{p}}_{i+1}$ are considered as the corresponding neighbouring points (spheres) in the sequence determining the skin; see Fig. 8 (top, left), with $\mathbf{p}_{-1}=\mathbf{p}_{n}$. First, we choose the tangent planes $\bar{\alpha}_{i}$ at $\overline{\mathbf{p}}_{i}$. These can be naturally determined by the points $\overline{\mathbf{p}}_{i}, \overline{\mathbf{p}}_{i-1}, \overline{\mathbf{p}}_{i+1}$, i.e., by the points $\overline{\mathbf{p}}_{i}$ and the tangent vectors

$$
\begin{array}{ll}
\overline{\mathbf{t}}_{i 1}=\lambda_{i 1}\left(\overline{\mathbf{p}}_{i-1}-\overline{\mathbf{p}}_{i}\right)=\left(\mathbf{t}_{i 1}, \tau_{i 1}\right), & \lambda_{i 1} \in \mathbb{R} ; \\
\overline{\mathbf{t}}_{i 2}=\lambda_{i 2}\left(\overline{\mathbf{p}}_{i+1}-\overline{\mathbf{p}}_{i}\right)=\left(\mathbf{t}_{i 2}, \tau_{i 2}\right), & \lambda_{i 2} \in \mathbb{R} ;
\end{array}
$$

see Fig. 8 (top, right). We note that a different method for choosing these tangent vectors can be employed when necessary. In particular, when some other part of the skin should be joined to the branched skin at a particular sphere, the tangents (at the corresponding point) are usually chosen to form a sufficiently small angle, cf. the inner spheres in Figs. 1, 10, and 14. In complex cases, designers' intervention may be necessary to obtain satisfactory results; see also Section 6.

Next, using the approach described in Section 3.1 we interpolate the points $\overline{\mathbf{p}}_{i}$ and the tangent planes $\bar{\alpha}_{i}$ by an RE surface $\overline{\mathbf{y}}(u, v)$. In particular we construct $\mathbf{x}(u, v)=$ $\mathbf{x}^{+}(u, v)$ and compute the function $f(u, v)$ and the medial surface $\mathbf{y}(u, v)$. Then the rational parametrization of 


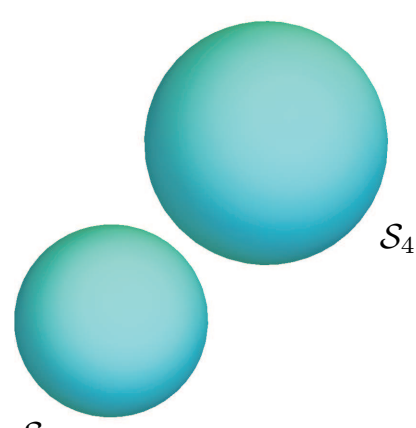

$\mathcal{S}_{1}$

$\mathcal{S}_{2}$
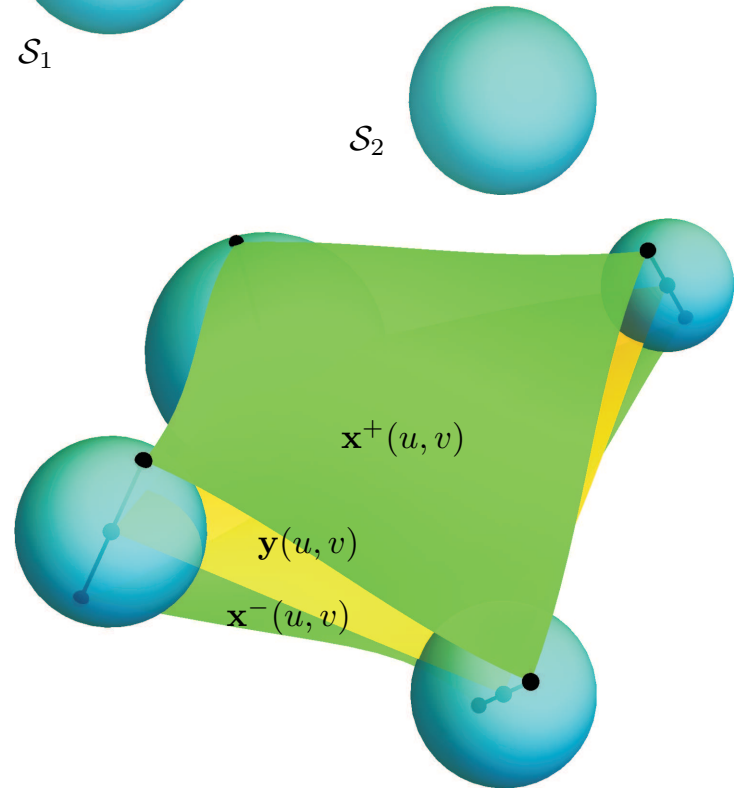
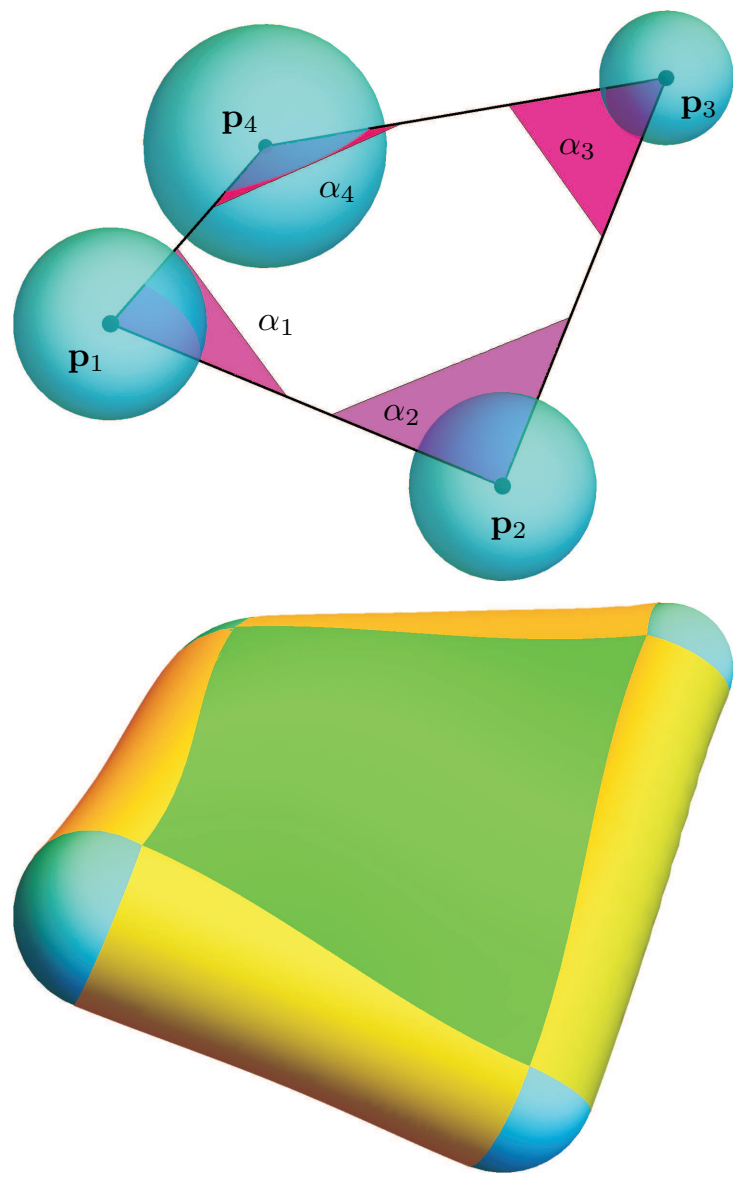

Figure 8: An illustration of the construction of the rational skinning surface (bottom right) between four given spheres (top left).

the second branch $\mathbf{x}^{-}(u, v)$ of the envelope surface can be easily obtained as

$$
\mathbf{x}^{-}(u, v)=\mathbf{x}^{+}+2 f \frac{\left(\mathbf{y}_{u} \times \mathbf{y}_{v}\right) \cdot\left(\mathbf{x}_{u} \times \mathbf{x}_{v}\right)}{\left|\mathbf{x}_{u} \times \mathbf{x}_{v}\right|^{2}}\left(\mathbf{y}_{u} \times \mathbf{y}_{v}\right)
$$

see Fig. 8 (bottom, left).

The whole skinning surface is composed of two branches of the envelope surface $\mathbf{x}^{ \pm}(u, v)$ and $n$ canal surfaces, cf. Fig. 8 (bottom, right), whose rational description can be computed by rotating the boundary curves $\mathbf{x}_{i}(u)$ of $\mathbf{x}(u, v)$ around the tangents of the boundary curves $\mathbf{y}_{i}(u)$ of $\mathbf{y}(u, v)$, cf. (2).

Remark 4.1. When constructing $\mathbf{x}(u, v)$ it may be convenient to prescribe not only the normal vectors $\mathbf{n}_{i}$ at the points $\mathbf{q}_{i}$ but also the tangent vectors which should be respected by the boundary curves. This is more suitable for modelling and influencing the output shape of the constructed patch. The tangent vectors $\mathbf{u}_{i j}, j=1,2$ can be chosen e.g. by projecting $\mathbf{t}_{i j}$ to the plane given by the point $\mathbf{q}_{i}$ and the normal vector $\mathbf{n}_{i}$, cf. (20). Then we construct a polynomial patch $\mathbf{x}(u, v)$ interpolating points $\mathbf{q}_{i}$ and tangent vectors $\mathbf{u}_{i j}$.

The construction of the skinning surface which smoothly envelopes 3 spheres is trivial and we mention it only for the sake of completeness. We can construct a triangular linear MST in the form

$\overline{\mathbf{y}}(u, v)=\overline{\mathbf{p}}_{2} u+\overline{\mathbf{p}}_{3} v+\overline{\mathbf{p}}_{1}(1-u-v), \quad u \in[0,1], v \in[0,1-u]$.

Since the points $\mathbf{p}_{i}, i=1,2,3$ lie in a common plane then both branches of the envelope are also linear (parts of planes); see Fig. 9 (left). Moreover, the boundary canal surfaces are cones; see Fig. 9 (right).

Since four (or more) points do not generically lie in one plane, we cannot use the simple approach for $n=3$ described above. Hence for $n>3$ we proceed according to the above described RE-method for constructing rational skinning surfaces smoothly joining $n$ prescribed spheres. Here we consider $n=4$.

The method proceeds as described in Section 3, and for constructing $\mathbf{x}(u, v)$ and $f(u, v)$ we employ Ferguson patches. The rational parametrization of the boundary canal surface can be obtained by (2), where

$$
\begin{array}{ll}
\mathbf{x}_{1}(u)=\mathbf{x}(0, u), & \mathbf{x}_{2}(u)=\mathbf{x}(u, 1), \\
\mathbf{x}_{3}(u)=\mathbf{x}(1, u), & \mathbf{x}_{4}(u)=\mathbf{x}(u, 0)
\end{array}
$$



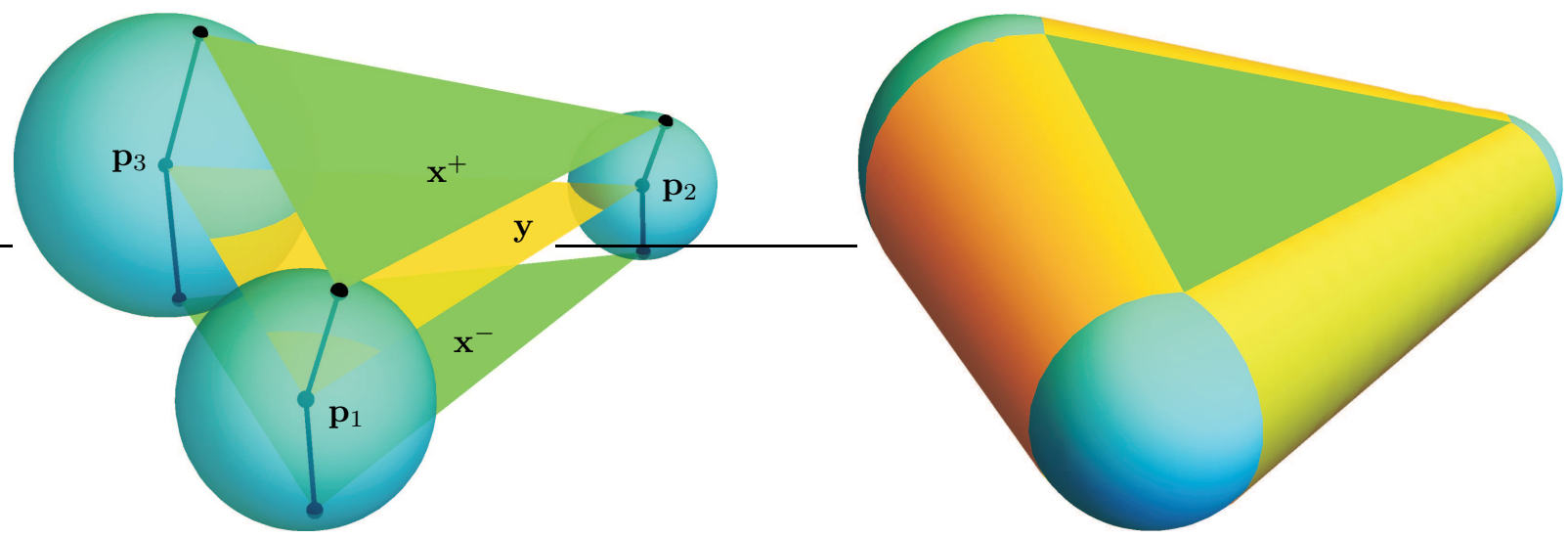

Figure 9: An illustration of the construction of the skinning surface between three spheres.
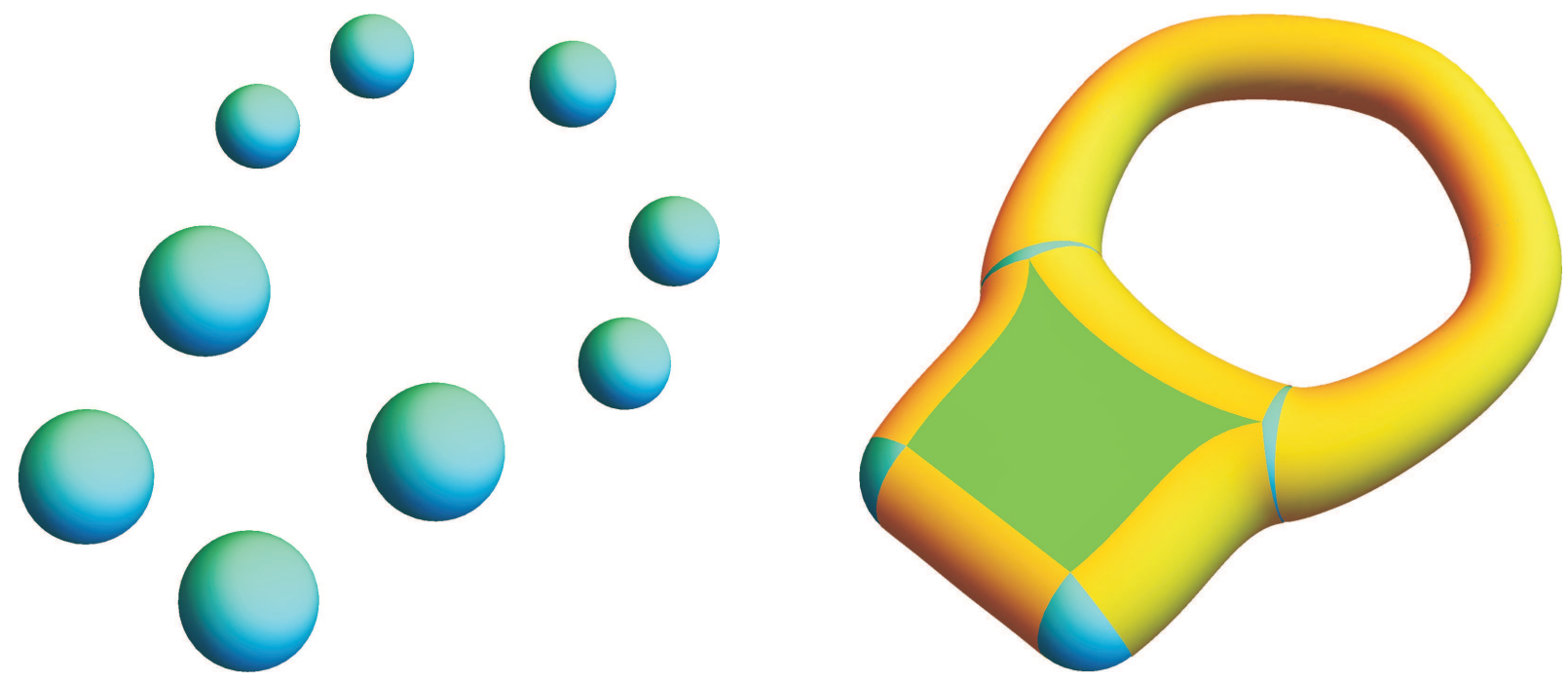

Figure 10: Construction of the rational branched skin of nine spheres

and

$$
\begin{array}{ll}
\mathbf{y}_{1}(u)=\mathbf{y}(0, u), & \mathbf{y}_{2}(u)=\mathbf{y}(u, 1), \\
\mathbf{y}_{3}(u)=\mathbf{y}(1, u), & \mathbf{y}_{4}(u)=\mathbf{y}(u, 0)
\end{array}
$$

and $\mathbf{y}(u, v)$ is computed using (11). The example in Fig. 8 was constructed this way.

Our method also allows us to construct branched skins of families of spheres. One such example is illustrated in Fig. 10. It is assumed that the connectivity (topology) of the skin is given, cf. [24].

\section{Blending between canal surfaces using rational envelope surfaces}

We now describe a method for computing blending surfaces smoothly joining $n \geq 3$ given canal surfaces. The method can be modified also for the construction of branched blends. For the sake of simplicity we replace the input canal surfaces by their tangent cones at the corresponding end circles. Indeed, these $n$ cones are represented in $\mathbb{R}^{3,1}$ by their linear MATs, i.e., as the lines

$$
\mathcal{C}_{i}: \overline{\mathbf{h}}_{i}(s)=\overline{\mathbf{p}}_{i}-s \overline{\mathbf{t}}_{i}, \quad i=1, \ldots, n,
$$

where the point $\overline{\mathbf{p}}_{i}$ corresponds to the sphere yielding the prescribed end circle; see Fig. 11 (top, left). Again we make use of RE surfaces, in particular we proceed according to the method presented in Section 3.2 for interpolating points and preserving tangent directions at boundaries. The end points $\overline{\mathbf{p}}_{i} \in \mathbb{R}^{3,1}$ and tangent vectors $\overline{\mathbf{t}}_{i} \in \mathbb{R}^{3,1}$ form the input of the algorithm.

We construct an RE surface interpolating $\overline{\mathbf{p}}_{i}$ and $\overline{\mathbf{t}}_{i}$; see Fig. 11 (top, right). The tangent planes $\bar{\alpha}_{i}$ were determined as

$$
\bar{\alpha}_{i}: \overline{\mathbf{p}}_{i}, \overline{\mathbf{t}}_{i}, \overline{\mathbf{p}}_{i+1}-\overline{\mathbf{p}}_{i-1}
$$

Then we compute the points $\mathbf{q}_{i}$ and the tangent vectors $\mathbf{u}_{i 1}, \mathbf{u}_{i 2}$ and construct a patch interpolating $\mathbf{q}_{i}$ and $\mathbf{u}_{i 1}, \mathbf{u}_{i 2}$ and having the second derivatives at the end points as free parameters. Solving (24) and (25) yields the parametric description of the medial surface $\mathbf{y}(u, v)$. The second 

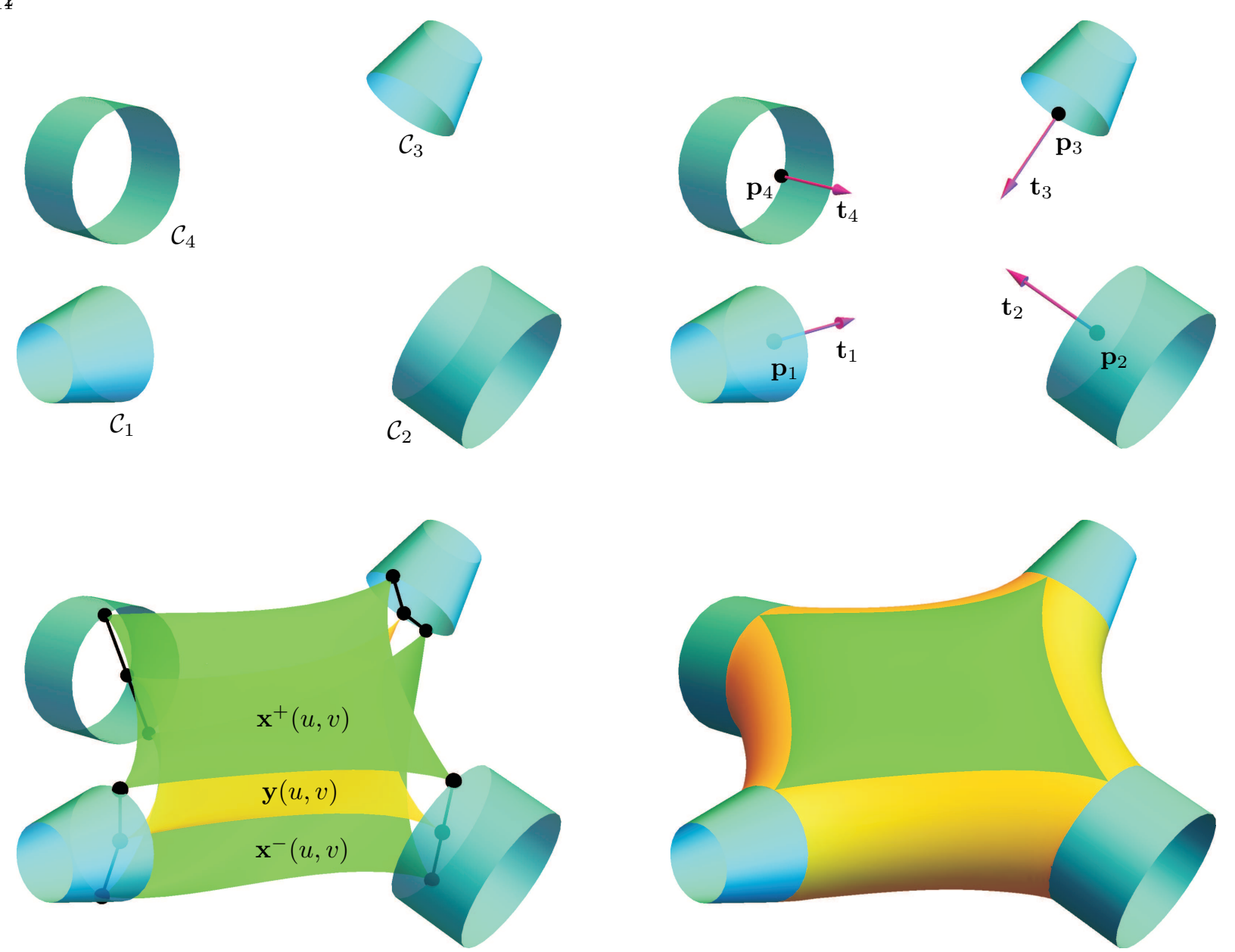

Figure 11: An illustration of the construction of a rational blending surface between four given cylinders and/or cones.

branch of the envelope is computed by (28), see Fig. 11 (bottom, left), and the boundary canal surfaces are obtained by (2), see Fig. 11 (bottom, right).

We emphasize that the choice of suitable points $\mathbf{q}_{i}$ is not fully automatic and can be guided by the designer; see Section 6 for a further discussion.

We now present examples for various situations and values of $n$. Consider (parts of) three cones given in $\mathbb{R}^{3,1}$ by the end points and tangent vectors

$$
\overline{\mathbf{p}}_{i}, \quad \overline{\mathbf{t}}_{i}, \quad i=1,2,3
$$

determining their MATs. We start by choosing tangent planes $\bar{\alpha}_{i}$ at $\overline{\mathbf{p}}_{i}$, i.e.,

$$
\bar{\alpha}_{i}: \overline{\mathbf{p}}_{i}, \overline{\mathbf{t}}_{i}, \overline{\mathbf{p}}_{j}-\overline{\mathbf{p}}_{k}, \quad i, j, k=1,2,3, i \neq j \neq k .
$$

After computing the envelope points $\mathbf{q}_{i}$, cf. (12), and choosing the tangent vectors $\mathbf{u}_{i 1}, \mathbf{u}_{i 2}$ at the tangent planes given by $\mathbf{q}_{i}$ and the normal vectors (13), we construct a quintic Bézier triangle interpolating the first-order data. The second derivatives at the corners $\mathbf{q}_{i}$ of the patch are kept as free parameters. Next, solving the linear systems
(24) and (25) with $\mathbf{t}_{i 1}=\mathbf{t}_{i 2}=\mathbf{t}_{i}$ yields the conditions for the first derivatives of the lifting function $f(u, v)$, which can be constructed as a cubic Bézier triangle. Then we construct the medial surface $\mathbf{y}(u, v)$, the second branch of the envelope surface (Fig. 12, left), and the boundary canal surfaces; see Fig. 12 (right).

In the case of four (parts of) cones/cylinders that should be blended we proceed according to the method described above. However now we employ a biquintic Bézier patch for constructing the surface $\mathbf{x}(u, v)$. For interpolating the values $f_{i}, f_{u}^{i}$ and $f_{v}^{i}$, obtained as a solution of (24) and (25), we use a Ferguson surface. Examples of this kind of blending are illustrated in Fig. 13.

Further, we can also use our method to combine skinning and branching in various ways. Such an example is shown in Fig. 1.

Yet another example uses a skinning surface consisting of two (one triangular and one quadrangular) patches and several canal surfaces constructed using the above described method (for blends). For the inner sphere, the exact tangent vectors (not only tangent plane) have to be prescribed, see Fig. 14. 

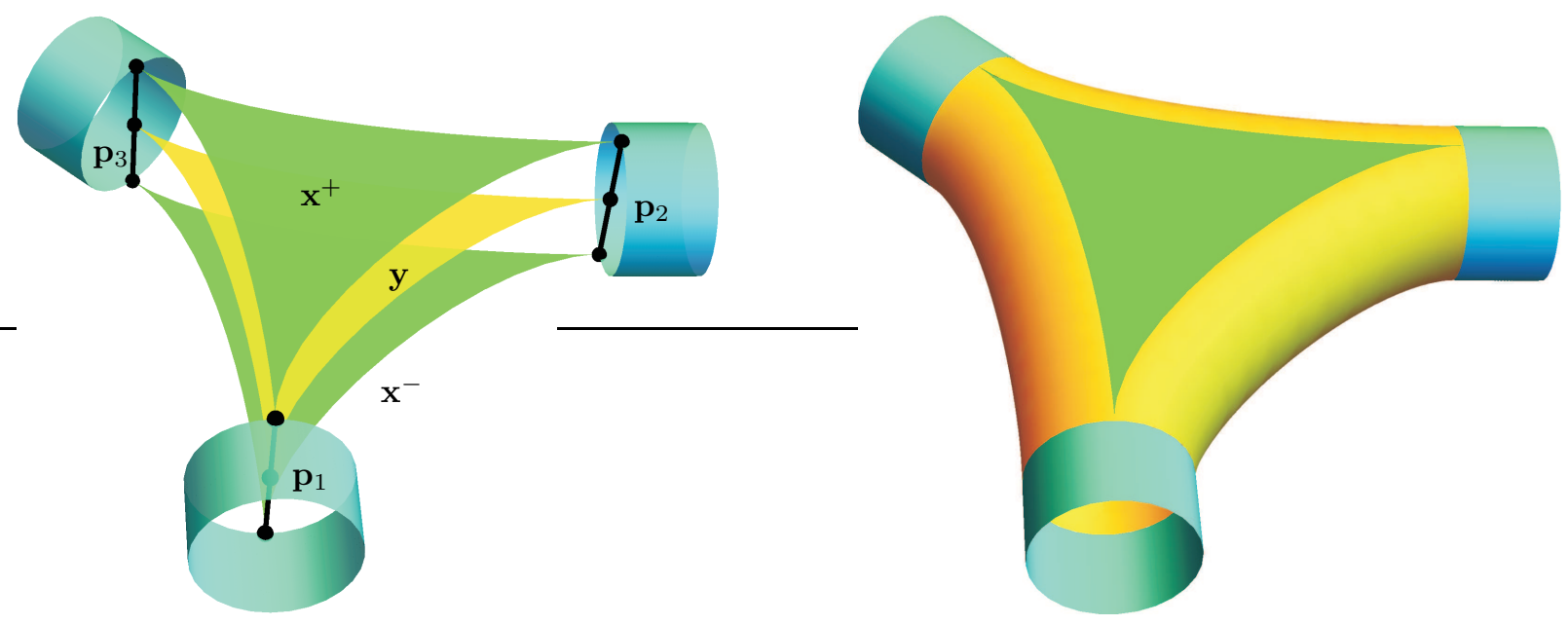

Figure 12: An illustration of the construction of the blending surface between three cylinders (cones).
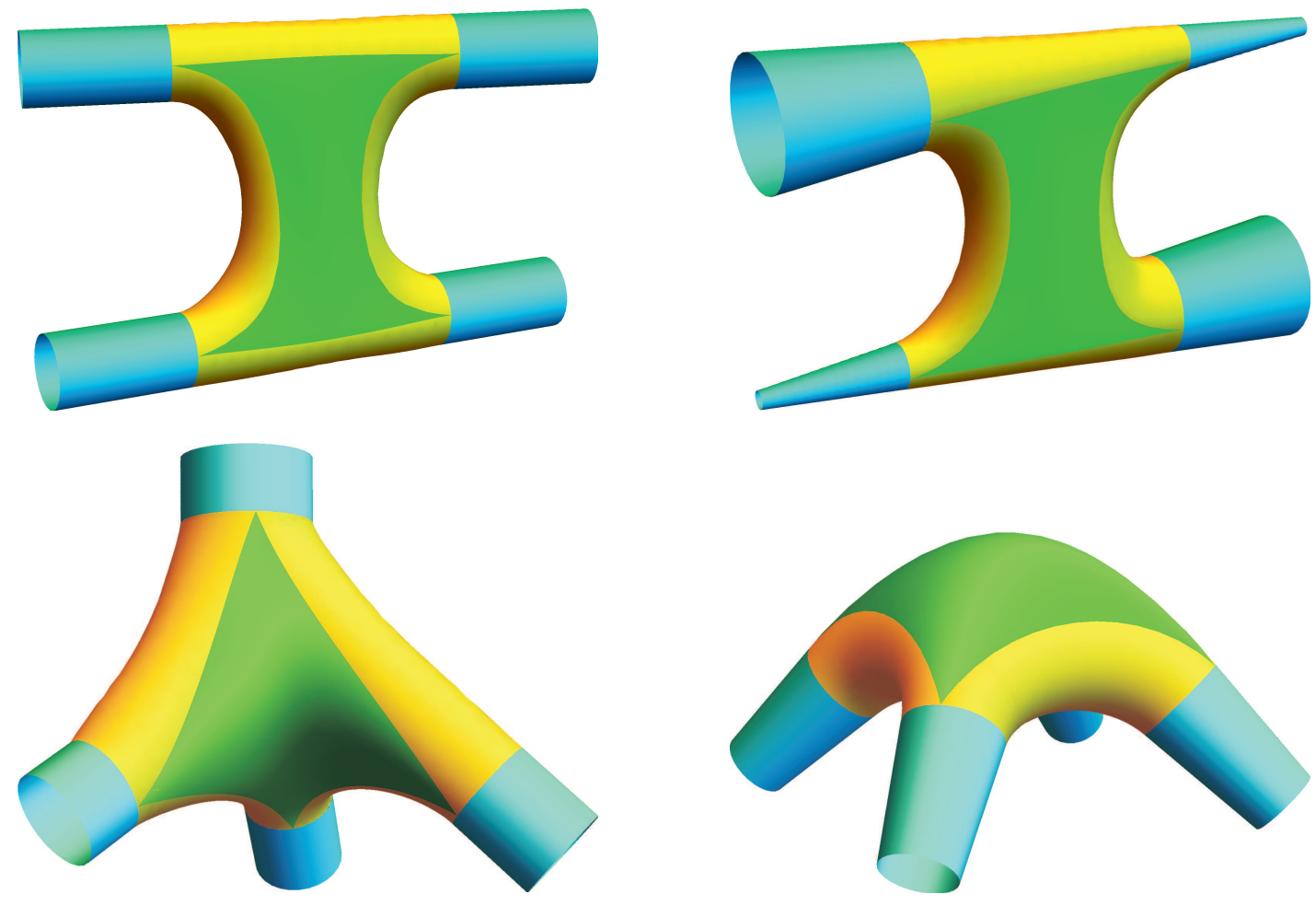

Figure 13: Construction of the rational blending surface between four given cylinders (cones)

\section{Limitations}

As already mentioned above, some of the choices of contact points and tangent vectors (or their magnitudes) depend on the designer's intent, and poor choices may lead to undesirable intersections in the resulting surface. This is directly analogous to the planar situation with circles/discs, as discussed for instance in [24].
One such unwanted intersection of a piece of the envelope surface with the input sphere is shown in Fig. 15, left. However, the RE surfaces employed for constructing skins and blends admit several modelling parameters (e.g. tangent vectors), and these can be suitably chosen such that intersections are avoided. In the current version of our algorithm, some constellations of the input shapes require the designer's intervention to obtain a satisfactory solu- 

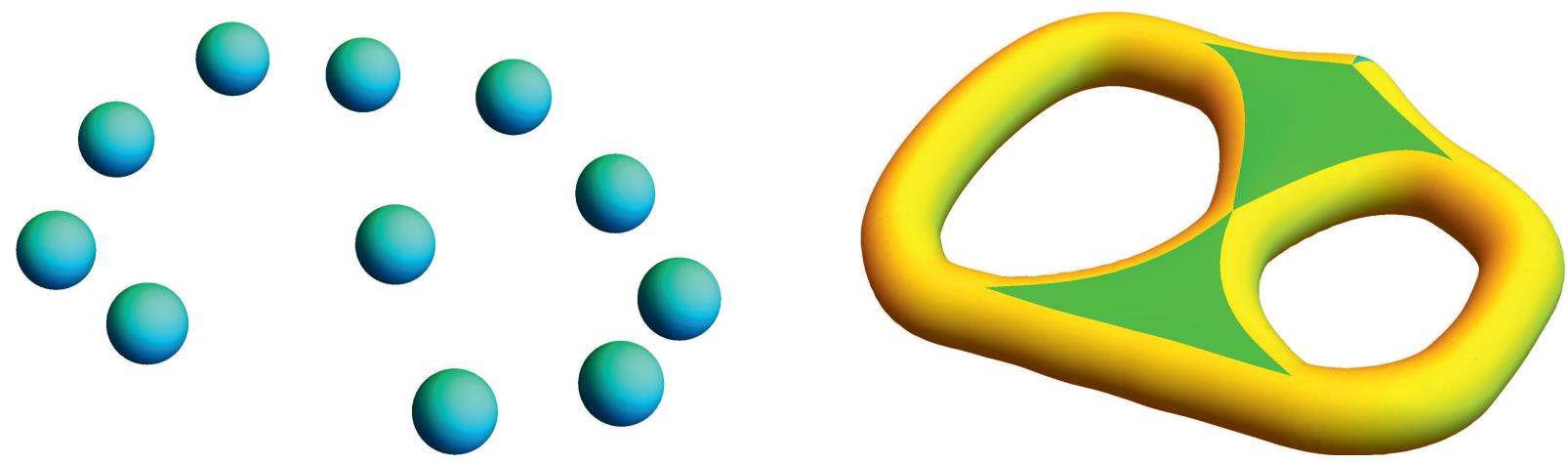

Figure 14: Construction of the rational branched skin of eleven spheres
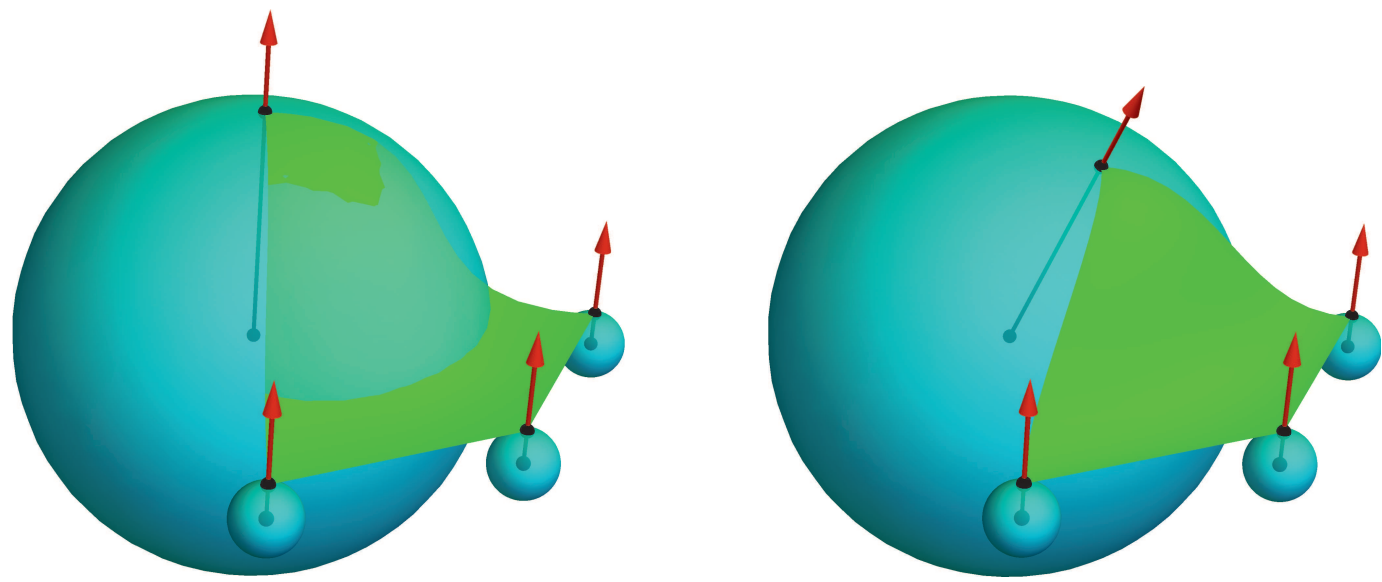

Figure 15: A problematic configuration of input spheres. Left: One branch of the envelope surface intersects one of the prescribed spheres. Right: The original intersection has been avoided by moving one of the contact points (top).

tion. This is shown in Fig. 15, right, where a modification of the prescribed tangent point (and thus also of the normal vector) was performed to obtain an intersection-free envelope.

Experience shows that it is necessary to avoid unnecessary twists of the constructed medial surface and the envelope. This then also maximizes the chance of obtaining not only a skin with no intersections with the input shapes, but also a skin with no singularities.

An appropriate and automatic determination of these points and vectors is a challenging avenue for future research.

\section{Conclusion}

We have investigated RE surfaces in detail and designed an algorithm for data interpolation using RE patches. These data include position and first derivatives at RE patch corners. The simplicity of our construction lends itself to higher-order Hermite data interpolation with RE surfaces.

To demonstrate the utility of our theoretical results, we have applied our method to two problems of practical importance: sphere skinning and canal surface blending. The resulting skins and blends are rational and allow us to smoothly skin or blend several input shapes.

It has not escaped our notice that our approach is not limited to $n$ equal to 3 or 4 . In the case when $n>4$, the principles of the skinning and blending constructions remain the same, but the goal is to construct an $n$-sided $\mathrm{RE}$ patch which interpolates, at its corners, $n$ points and their associated normals.

To this end, one could employ constructions that rely on generalized barycentric coordinates such as those of $[32,33]$ and construct a single patch satisfying the interpolation conditions. However, we believe it might be preferable to employ constructions that are fully compatible with standard CAD systems, such as the variants [34] of Clough-Tocher splines [35] or other spline constructions $[36,37,38]$, where the parametric $n$-gon needs to be decomposed into $n-2$ macro-triangles.

\section{Acknowledgements}

Michal Bizzarri and Miroslav Lávička were supported by the project LO1506 of the Czech Ministry of Educa- 
tion, Youth and Sports. We thank to all referees for their valuable comments, which helped us to improve the paper.

\section{References}

[1] Gerald Farin. Curves and Surfaces for Computer-Aided Geometric Design. Academic Press, 1988.

[2] Les Piegl and Wayne Tiller. The NURBS book. Monographs in Visual Communication. Springer-Verlag New York, Inc., 2 edition, 1997.

[3] J. Hoschek and D. Lasser. Fundamentals of Computer Aided Geometric Design. AK Peters, 1993.

[4] R.T. Farouki. Pythagorean-Hodograph Curves: Algebra and Geometry Inseparable. Springer, 2008.

[5] Robin Hartshorne. Algebriac Geometry. Springer-Verlag, 1977.

[6] B. Bastl, B. Jüttler, M. Lávička, T. Schulz, and Z. Sír. On the parameterization of rational ringed surfaces and rational canal surfaces. Mathematics in Computer Science, 8(2):299319, 2014.

[7] Michal Bizzarri and Miroslav Lávička. On modeling with rational ringed surfaces. Computer-Aided Design, 58:151-161, 2015. Solid and Physical Modeling 2014.

[8] Michal Bizzarri, Miroslav Lávička, and Jiř́ Kosinka. Medial axis transforms yielding rational envelopes. Computer Aided Geometric Design, 46:92-102, 2016.

[9] H.P. Moon. Minkowski Pythagorean hodographs. Computer Aided Geometric Design, 16:739-753, 1999.

[10] J. Kosinka and M. Lávička. On rational Minkowski Pythagorean hodograph curves. Computer Aided Geometric Design, 27(7):514-524, 2010.

[11] J. Kosinka and B. Jüttler. MOS surfaces: Medial surface transforms with rational domain boundaries. In The Mathematics of Surfaces XII, volume 4647 of Lecture Notes in Computer Science, pages 245-262. Springer, 2007.

[12] Pan Li, Bin Wang, Feng Sun, Xiaohu Guo, Caiming Zhang, and Wenping Wang. Q-MAT: Computing medial axis transform by quadratic error minimization. ACM Trans. Graph., 35(1):8:18:16, December 2015.

[13] B. Bastl, B. Jüttler, M. Lávička, and T. Schulz. Blends of canal surfaces from polyhedral medial surface transform representations. Computer-Aided Design Design, 43(11):1477-1484, 2011.

[14] Martin Peternell and Helmut Pottmann. Computing rational parametrizations of canal surfaces. Journal of Symbolic Computation, 23:255-266, February 1997.

[15] Günter Landsmann, Josef Schicho, and Franz Winkler. The parametrization of canal surfaces and the decomposition of polynomials into a sum of two squares. Journal of Symbolic Computation, 32(1-2):119-132, 2001.

[16] Michal Bizzarri and Miroslav Lávička. A symbolic-numerical method for computing approximate parameterizations of canal surfaces. Computer-Aided Design, 44(9):846 - 857, 2012.

[17] Simon L. Altmann. Rotations, quaternions, and double groups. Dover Publications, 2005.

[18] Ron Goldman. Understanding quaternions. Graphical Models, 73:21-49, March 2011.

[19] Gerald Farin. Curves and surfaces for CAGD: A practical guide. Morgan Kaufmann Publishers Inc., San Francisco, CA, USA, 2002.

[20] G. Slabaugh, B. Whited, J. Rossignac, T. Fang, and G. Unal. 3D ball skinning using PDEs for generation of smooth tubular surfaces. Computer-Aided Design, 42(1):18-26, 2010.

[21] R. Kunkli and M. Hoffmann. Skinning of circles and spheres. Computer Aided Geometric Design, 27(8):611-621, 2010.

[22] Kornél Bana, Kinga Kruppa, Roland Kunkli, and Miklós Hoffmann. KSpheres - an efficient algorithm for joining skinning surfaces. Computer Aided Geometric Design, 31(7-8):499-509, 2014.

[23] M. Hoffmann, J. Monterde, and E. Troll. Blending of spheres by rotation-minimizing surfaces. Journal for Geometry and Graphics, 19(2):189-200, 2015.
[24] Bohumír Bastl, Jiří Kosinka, and Miroslav Lávička. Simple and branched skins of systems of circles and convex shapes. Graphical Models, 78:1-9, 2015.

[25] Michal Bizzarri and Miroslav Lávička. Parameterizing rational offset canal surfaces via rational contour curves. ComputerAided Design, 45(2):342-350, 2013.

[26] Paul Yushkevich, P. Thomas Fletcher, Sarang Joshi, Andrew Thall, and Stephen M. Pizer. Continuous medial representations for geometric object modeling in $2 \mathrm{D}$ and $3 \mathrm{D}$. Image and Vision Computing, 21(1):17 - 27, 2003.

[27] Helmut Pottmann. Rational curves and surfaces with rational offsets. Computer Aided Geometric Design, 12(2):175-192, 1995.

[28] Jiří Kosinka and Miroslav Lávička. A unified Pythagorean hodograph approach to medial axis transform and offset approximation. Journal of Computational and Applied Mathematics, 235(12):3413-3424, 2011.

[29] Richard W. Daniels. Introduction to Numerical Methods and Optimization Techniques. Elsevier Science Ltd, 1978.

[30] Arnold Neumaier. Introduction to Numerical Analysis. Cambridge University Press, 2001.

[31] Victor S. Ryaben'kii and Semyon V. Tsynkov. A Theoretical Introduction to Numerical Analysis. Chapman and Hall/CRC, 2006.

[32] Xian-Ying Li, Tao Ju, and Shi-Min Hu. Cubic mean value coordinates. ACM Trans. Graph., 32(4):126:1-126:10, July 2013.

[33] Tamás Várady, Péter Salvi, and György Karikó. A multi-sided bézier patch with a simple control structure. Computer Graphics Forum, 35(2):307-317, 2016.

[34] Jiří Kosinka and Thomas J. Cashman. Watertight conversion of trimmed $\{\mathrm{CAD}\}$ surfaces to clough-tocher splines. Computer Aided Geometric Design, 37:25 - 41, 2015.

[35] R. W. Clough and J. L. Tocher. Finite element stiffness matrices for analysis of plates in bending. In Conference on Matrix Methods in Structural Mechanics, pages 515-545. Wright Patterson Air Force Base, Ohio, 1965.

[36] Tianjun Wang. A $C^{2}$-quintic spline interpolation scheme on triangulation. Computer Aided Geometric Design, 9(5):379386, November 1992.

[37] Peter Alfeld. A bivariate $C^{2}$ Clough-Tocher scheme. Computer Aided Geometric Design, 1(3):257-267, December 1984.

[38] Hendrik Speleers. Interpolation with quintic Powell-Sabin splines. Applied Numerical Mathematics, 62(5):620-635, May 2012. 